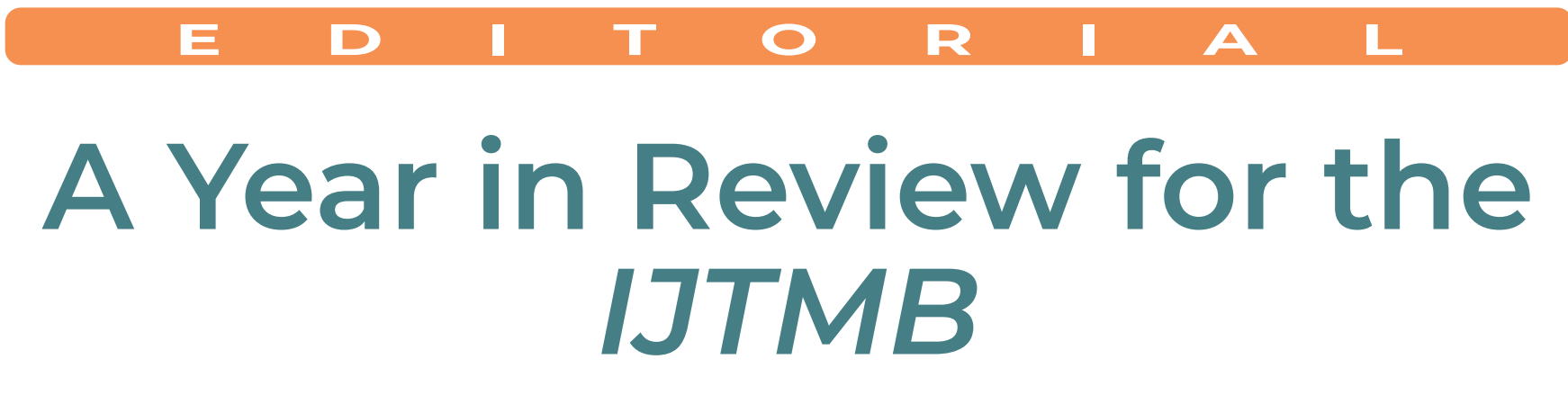

Ann Blair Kennedy, LMT, BCTMB, DrPH, Executive Editor/Editor-in-Chief, IJTMB

University of South Carolina School of Medicine Greenville, Greenville, SC, USA

https://doi.org/10.3822/ijtmb.v75i1.735

Yearly review and evaluation of the journal metrics and processes help to understand the value, worth, and impact of the Journal. In this editorial, the yearly review includes discussions on the current statistics of journal submissions and publication, updates to the IJTMB website, current social media impact, as well as the current peer review process and metrics. Additionally, the peer reviewers for the past year are thanked and acknowledged for their efforts and service to the Journal.

KEYWORDS: peer review; publishing; publications; writing; quality improvement

\section{A Year in Review}

Evaluation science helps us to understand the worth, value, context, and significance of a policy, person/group, program, or intervention. (1) Regular evaluation can help guide decisions, identify areas of improvements, and help improve processes with these areas. As Executive Editor/Editor-in-Chief of the IJTMB , it is important for me to review, evaluate, and report the findings to the stakeholders. Therefore, in this editorial I will explore the current evaluation and improvements from December 1, 2020 through November 29, 2021.

\section{Current Statistics}

Over the time mentioned above, the IJTMB received 36 manuscript submissions (24 research, 8 practice, 2 education, and 2 commentaries), and a total of 16 submissions were accepted with 23 submissions declined (9 desk reject, 14 after peer review). The number of accepted/ declined manuscripts does not match the number submitted during this time frame as some of these acceptance/declines were already in the peer review pipeline prior to the evaluation period. Desk rejections are those that are rejected prior to being sent out to peer review. In general this happens when the manuscript does not fit the aims and scope of the Journal and/or is not written for the IJTMB readership. Four issues were published (March, June, September, and December) with a total of 13 articles included in those issues.

In last year's evaluation of trends in publishing education and practice content, (2) it was noted that over the two previous years, the IJTMB published 5\% and $17 \%$ content in the Education and Practice sections, respectively. That editorial called for more submissions for these sections. (2) Comparatively, while submissions to the Practice section are slightly increased to 20\%, submissions to the Education section remain at $5 \%$ of all submissions to the Journal in the one year time frame. However, no education manuscripts were published last year and only two papers (15\%) were published in the Practice section. Therefore, we will repeat the call from the previous editorial, (2) and encourage and request educators and practitioners to submit manuscripts for these sections.

\section{Website Improvements and New Metrics for Authors}

This past year also allowed for improvements to the IJTMB website including enhanced interface and navigation, as well as the incorporation of tools and widgets to help authors track their impact. The upgrade to Open Journal Systems (OJS) 3.2 is mostly useful for those who manage the Journal and work with the authors and reviewers; however, this upgrade does include some reader-enhancement tools. Readers and authors can now see a) the articles 
that are most read in the past seven days, b) the number of article views/downloads for a given paper in the past 12 months (Figure 1), and c) article-sharing tools that allow the reader to share the article to social media and via email (Figure 1). Additional alternative metrics have also been added to the site so that authors can help track the impact of their research more completely than only by number of citations their article received. Figure 1 shows these new metrics and reader-enhancement tools for the most viewed and downloaded article for the evaluation year. ${ }^{(3)}$

\section{Social Media}

The IJTMB Facebook page continues to share not only the current content published by the Journal, but also new and current massage therapy research by other publishers. Currently the page has over 3,000 followers, with a majority of the audience from the United States (53.8\%) and Canada (20.5\%). A majority of the followers are women (81.3\%) and are between the ages of 35-54 (62.1\%). In reviewing the page reach, there was a $29 \%$ increase over the year evaluation period, with marked spikes when a new issue is published quarterly.

\section{Peer Review Evaluation and Appreciation for Peer Reviewers}

A previous editorial last year evaluated the peer review process at the IJTMB and provided some insights and calls to action. (4) The evaluation of the peer review process this year revealed that approximately $37.3 \%$ of people invited to peer review do not respond to the invitation. With more than a third of reviewers not responding to invitations, this increases the time it can take to go through the process. Authors often contact the editors during the peer review for updates on their paper's progress through the process. Understanding the potential time frame for the peer review process can help authors prior to submitting to any journal. Consequently, it is important to report the statistics for acceptance and rejection of papers that have been submitted. From December 1, 2020 to November 29, 2021, it took on average 4.3 months (mean=133 days, SD 66.4) days to accept papers submitted during this time frame. The length of time to accept a paper includes the peer review process and time for the authors to revise their papers based on peer review and editorial comments. On average, including desk rejected papers, papers were rejected in just over a month
The Effects of Self-Massage on Osteoarthritis of the Knee: a Randomized, Controlled Trial
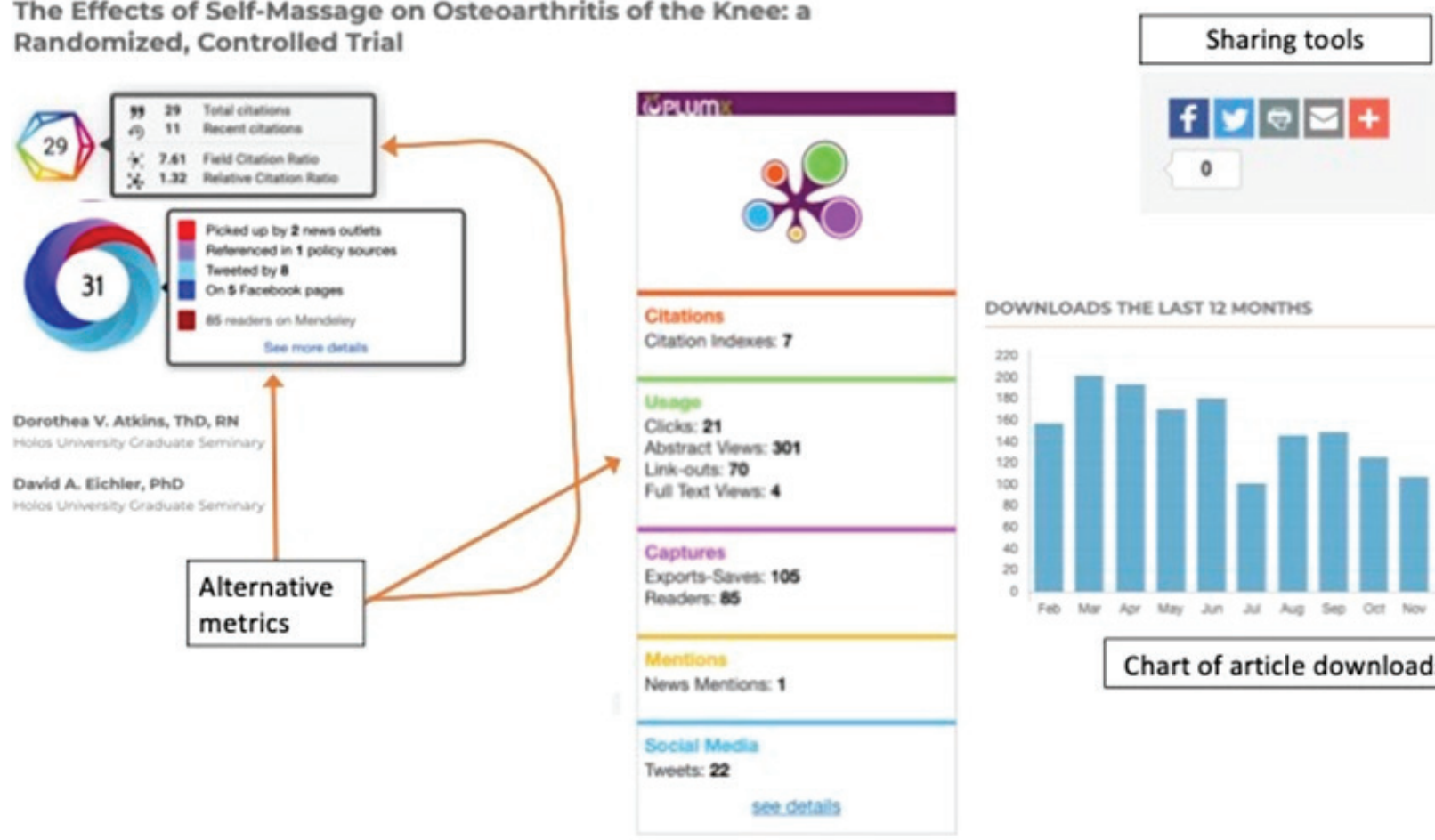

f부무+ 0

DOWNLOADS THE LAST 12 MONTHS

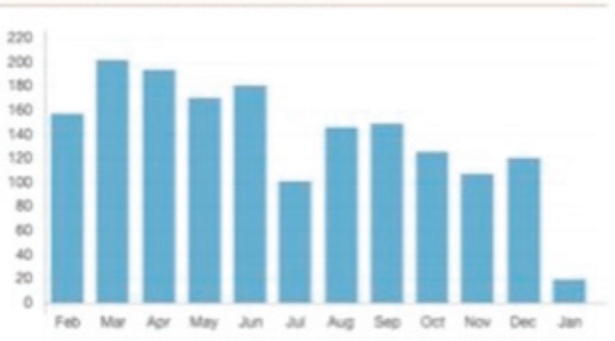

Chart of article downloads

FIGURE 1. Reader enhancement tools and alternative metrics for articles 
and a half (mean=43.9 days, SD 65.32); excluding the desk rejections, the amount of time increases to 2.8 months (mean=83.86 days, SD 74.14).

In further reviewing the peer review process for the time frame indicated, a total of 45 individuals provided their expertise as peer reviewers and 16 of those people reviewed two or more manuscripts. However, to complete those reviews, we invited a total of 153 to peer review, revealing that only $43 \%$ who are invited actually completed the review. In the previous evaluation, 51\% completed reviews (Figure 2). (4) Additionally, compared to the last evaluation, we now have a higher percentage of individuals not responding to the invitation (37\%) to peer review compared to the previous evaluation (27.8\%). ${ }^{(4)}$ More than half (53\%) of those invited to peer review either do not respond to the invitation or decline the invitation, which is an increase from the previous evaluation (48.7\%). (4) Considering the progress of the Covid-19 pandemic, it is understandable that there was this difficulty in recruiting peer reviewers. Other journals and societies have reported similar findings of having difficulty in recruiting peer reviewers, and researchers have also been reporting difficulty finding time to peer review during the pandemic. ${ }^{(5-7)}$

Peer reviewers are vital to any scientific journal process, and while we do offer an incentive of three CEs for completing their review in a timely manner, we must still show appreciation for those who offered

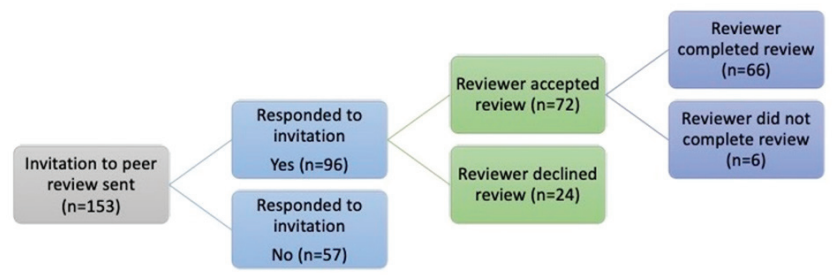

FIGURE 2. Flow of peer review from invitation to review completion their expertise as a peer reviewer. $(4,8)$ Thank you peer reviewers; we truly appreciate your time and the expertise you provided. Below are the names of those who were peer reviewers within the oneyear time frame; those who reviewed two or more times are identified by an * next to their name.
Nityal Kumar Alagingi Derek Richard Austin* John J Balletto Amanda Baskwill* Leisa Bellmore* Oluwagbemiga Dadematthews

Robyn Dey*

Krisa Fairbanks

Leora Fellus*

Sarah Fogarty

Alexandra Forsythe Luann Drolc Fortune* Sandy Fritz Charlene Gaffney Cecilia Garrison Lauren Gonzales Donelda Gowan Kimberly A. Griffith Adam Hernandez* Younes Jahangiri Noudeh

Alisa Joy Johnson Arezou Karampourian
Alex Kidd

Cherie Kiesler

Brooke Martin

Suzanne Michaud*

Seyedmohammad

Mirhosseini*

Ronna Moore

Albert Moraska*

Gopal Nambi*

P Namiranian*

Frances Neal

Dennis Newhook

Mollie Newhouse

Megan O'Connor

Jose M. Orozco*

Stefanie Jo Pusateri*

Stephen Redmon*

Mica Rosenow

Christin Sadler*

Jeffrey Shuman

Robin Byler Thomas

Lori Thompson

Rebecca Torns-Barker

Gretchen Wetherby

\section{COPYRIGHT}

Published under the CreativeCommons Attribution-NonCommercial-NoDerivs 3.0 License.

Corresponding author: Ann Blair Kennedy, LMT, BCTMB, DrPH, University of South Carolina School of Medicine Greenville, 701 Grove Road, Greenville, SC 29605, USA

E-mail: ExecEditor@IJTMB.org 